\title{
Fisher and Shannon information from one-matrix. Link to the kinetic energy
}

\author{
Á. Nagy $^{a}$ and E. Romera ${ }^{b}$ \\ ${ }^{a}$ Department of Theoretical Physics, University of Debrecen, \\ H-4010 Debrecen, Hungary \\ e-mail:anagy@madget.atomki.hu, tel:36-52-509201 \\ ${ }^{b}$ Departamento de Física Atómica, Molecular y Nuclear \\ and Instituto Carlos I de Física Teórica y Computacional, \\ Universidad de Granada, Fuentenueva s/n, 18071 Granada, Spain \\ (Dated: February 4, 2014)
}

\begin{abstract}
A six-dimentional distribution function $F$ is constructed from the one-matrix of a non-interacting system. For double occupied orbitals and real one-matrix, the Fisher information $I_{F}$ constructed from the distribution function $F$ is proportional to the non-interacting kinetic energy $T_{s}$. A local six-dimensional wave-vector $q$ is defined that establishes a link between the Shannon and Fisher information and the local kinetic energy. Illustrative examples for Coulomb systems are presented.
\end{abstract}

\section{INTRODUCTION}

The link between information theory and quantum mechanical kinetic energy was first established by Sears, Parr and Dinur [1] three decades ago. They derived an expression for the kinetic energy

$$
T=\frac{1}{8} I_{n}+\frac{1}{8} \int n(\mathbf{r}) \tilde{i}_{f}(\mathbf{r}) d \mathbf{r}
$$

where the first term is proportional to Fisher information [2]

$$
I_{n}=\int \frac{|\nabla n|^{2}}{n} d \mathbf{r}
$$

constructed from the electron density $n$

$$
n(\mathbf{r})=N \int\left|\Psi\left(\mathbf{r}, \sigma_{1}, \mathbf{r}_{2}, \sigma_{2}, \ldots, \mathbf{r}_{N}, \sigma_{N}\right)\right|^{2} d \sigma_{1} d \mathbf{r}_{2} d \sigma_{2} \ldots d \mathbf{r}_{N} d \sigma_{N}
$$

and in the second term $\tilde{i}_{f}$ is a Fisher information density

$$
\tilde{i}_{f}(1)=\int \frac{\left[\nabla_{1} f(2, \ldots, N \mid 1)\right]^{2}}{f(2, \ldots, N \mid 1)} d 2 \ldots d N
$$

associated with the conditional density $f(2, \ldots, N \mid 1)=|\Psi|^{2} / n(\mathbf{r})$. $\Psi$ is the wave function. (They normalized the one-particle density to 1 . In this paper $n$ is normalized to $\mathrm{N}$ as is usually done in density functional theory. That is why we applied a different factor in Eq. (1).) The first term in Eq. (1) is the full Weizsäcker kinetic energy $T_{W}[3]$.

The relationship between the Fisher information and the kinetic energy has been discussed in several papers [48, 10, 11]. Ghiringhelli, Hamilton and Delle Site [13] proposed that the second term in Eq. (1) is proportional to the Shannon information [14].

$$
S_{n}=-\int n(\mathbf{r}) \ln n(\mathbf{r}) d \mathbf{r},
$$

constructed from the electron density $n$. Uncertainty-type lower bound to the Shannon information sum [15] to the entropy sum in complementary spaces was formulated by Gadre $[16,17]$. The upper and lower bounds for the configuration and momentum space entropies were expressed with the kinetic energy [18].

The relationship between the Fisher and Shannon information has been studied by several authors [11, 12, 19]. Fisher information can be defined using pair density [20] and generalized pair density functions [21], as well, leading to interesting relation to the kinetic energy. Generalized Weizsäcker functionals, that is, Fisher information obtained from two-electron, three-electron distribution functions have recently been studied by Chakraborty and Ayers [22]. They found that higher-order Weizsäcker functionals fail in approximating the total kinetic energy.

In Section 2 we show, that one can construct a six-dimentional distribution function $F$ from the one-matrix of the non-interacting system. In case we have double occupied orbitals and real one-matrix, the Fisher information $I_{F}$ constructed from the distribution function $F$ is proportional to the non-interacting kinetic energy $T_{s}$. In Section 3 a local six-dimensional wave-vector $q$ is defined that establishes a link between the Shannon and Fisher information and the local kinetic energy. Section 4 presents illustrations for Coulomb systems and discussion. 


\section{FISHER AND SHANNON INFORMATION FROM IDEMPOTENT ONE-MATRIX}

Consider a non-interacting system with the wave function $\Phi$. The one-matrix is defined as

$$
\gamma\left(\mathbf{r}, \mathbf{r}^{\prime}\right)=N \int \Phi^{*}\left(\mathbf{r}^{\prime}, \sigma_{1}, \mathbf{r}_{2}, \sigma_{2}, \ldots, \mathbf{r}_{N}, \sigma_{N}\right) \Phi\left(\mathbf{r}, \sigma_{1}, \mathbf{r}_{2}, \sigma_{2}, \ldots, \mathbf{r}_{N}, \sigma_{N}\right) d \sigma_{1} d \mathbf{r}_{2} d \sigma_{2} \ldots d \mathbf{r}_{N} d \sigma_{N}
$$

where $N$ is the number of electrons. If we have double occupied orbitals $\phi_{i}$, the one-matrix can also be written as

$$
\gamma\left(\mathbf{r}, \mathbf{r}^{\prime}\right)=2 \sum_{i=1}^{N / 2} \phi_{i}^{*}\left(\mathbf{r}^{\prime}\right) \phi_{i}(\mathbf{r})
$$

For orthonormal orbitals we obtain that the one-matrix is idempotent:

$$
\int \gamma\left(\mathbf{r}, \mathbf{r}_{2}\right) \gamma\left(\mathbf{r}_{2}, \mathbf{r}^{\prime}\right) d \mathbf{r}_{2}=2 \gamma\left(\mathbf{r}, \mathbf{r}^{\prime}\right)
$$

Define now a six-dimensional probability density as

$$
F\left(\mathbf{r}, \mathbf{r}^{\prime}\right)=\frac{1}{2 N}\left|\gamma\left(\mathbf{r}, \mathbf{r}^{\prime}\right)\right|^{2}
$$

$F$ is normalized to 1 as

$$
\int F\left(\mathbf{r}, \mathbf{r}^{\prime}\right) d \mathbf{r} d \mathbf{r}^{\prime}=\frac{1}{2 N} \int \gamma\left(\mathbf{r}^{\prime}, \mathbf{r}\right) \gamma\left(\mathbf{r}, \mathbf{r}^{\prime}\right) d \mathbf{r} d \mathbf{r}^{\prime}=\frac{1}{N} \int \gamma(\mathbf{r}, \mathbf{r}) d \mathbf{r}=1 .
$$

Introducing the six-dimensional vector $\mathbf{R}=\mathbf{r}, \mathbf{r}^{\prime}$ the Shannon and the Fisher information are defined as

$$
S_{F}=\int s_{F}(\mathbf{R}) d \mathbf{R}
$$

and

$$
I_{F}=\int i_{F}\left(\mathbf{r}, \mathbf{r}^{\prime}\right) d \mathbf{r} d \mathbf{r}^{\prime}=\int i_{F}(\mathbf{R}) d \mathbf{R}
$$

where

$$
s_{F}=-F(\mathbf{R}) \ln F(\mathbf{R})
$$

and

$$
i_{F}(\mathbf{R})=\frac{\left|\nabla_{R} F(\mathbf{R})\right|^{2}}{F(\mathbf{R})}
$$

are the Shannon and the Fisher information densities.

For real one-matrix, the Fisher information $I_{F}$ is proportional to the non-interacting kinetic energy $T_{s}$. Keeping in mind that the non-interacting kinetic energy $T_{s}$ can be written as

$$
T_{s}=\left.\frac{1}{2} \int \nabla_{r_{1}} \nabla_{r_{2}} \gamma\left(\mathbf{r}_{1}, \mathbf{r}_{2}\right)\right|_{\mathbf{r}_{2}=\mathbf{r}_{1}} d \mathbf{r}_{1}
$$

the idempotency condition (7) leads to

$$
\begin{aligned}
T_{s} & =\left.\frac{1}{4} \int \nabla_{r_{1}} \nabla_{r_{2}} \gamma\left(\mathbf{r}_{1}, \mathbf{r}_{3}\right) \gamma\left(\mathbf{r}_{3}, \mathbf{r}_{2}\right)\right|_{\mathbf{r}_{2}=\mathbf{r}_{1}} d \mathbf{r}_{1} d \mathbf{r}_{3} \\
& =\frac{1}{4} \int \nabla_{r_{1}} \gamma\left(\mathbf{r}_{1}, \mathbf{r}_{3}\right) \nabla_{r_{1}} \gamma\left(\mathbf{r}_{3}, \mathbf{r}_{1}\right) d \mathbf{r}_{1} d \mathbf{r}_{3} \\
& =\frac{1}{8} \int\left[\left(\nabla_{r_{1}} \gamma\left(\mathbf{r}_{1}, \mathbf{r}_{3}\right)\right)^{2}+\left(\nabla_{r_{3}} \gamma\left(\mathbf{r}_{1}, \mathbf{r}_{3}\right)\right)^{2}\right] d \mathbf{r}_{1} d \mathbf{r}_{3}
\end{aligned}
$$


Using the six-dimensional vector $\mathbf{R}$ the non-interacting kinetic energy $T_{s}$ takes the form

$$
T_{s}=\int \tau_{s}(\mathbf{R}) d \mathbf{R}
$$

where

$$
\tau_{s}(\mathbf{R})=\frac{1}{8}\left[\nabla_{R} \gamma(\mathbf{R})\right]^{2}
$$

is the local kinetic energy. Comparing Eqs. (9), (12),(17) and (18) we are led to the relation between the Fisher information $I_{F}$ and the non-interacting kinetic energy $T_{s}$ :

$$
I_{F}=\frac{16}{N} T_{s}
$$

and the corresponding local quantities:

$$
i_{F}=\frac{16}{N} \tau_{s}
$$

On the other hand, it is known that the moments of the density $F\left(\left\langle R^{a}\right\rangle=\int R^{a} F(\mathbf{R}) d \mathbf{R}\right)$ are related with the Fisher information as [23]

$$
I_{F} \geq(\beta+D-1)^{2} \frac{\left\langle r^{\beta-1}\right\rangle^{2}}{\left\langle r^{2 \beta}\right\rangle}, \quad \beta \geq \max \{-D+1,-1\}
$$

where $D$ is the dimension of the system. So if we take $D=6$ and $\beta=1$ we can write

$$
I_{F} \geq \frac{6^{2}}{\left\langle R^{2}\right\rangle} \text {. }
$$

Now, using equations (3) and (19) we obtain a bound between the non-interacting kinetic energy and the magnetic susceptibility

$$
T_{s} \geq \frac{3^{2}}{2^{3}} \frac{N^{2}}{\left\langle r^{2}\right\rangle}
$$

with $\left\langle r^{2}\right\rangle=\int r^{2} n(\mathbf{r}) d \mathbf{r}$. Writing the total non-interacting kinetic energy (Eq.(15)) as $T_{s}=\left\langle p^{2}\right\rangle / 2$ the uncertainty relation

$$
\left\langle p^{2}\right\rangle\left\langle r^{2}\right\rangle \geq \frac{9 N^{2}}{4}
$$

can be obtained.This inequality was derived earlier by Gadre and Chakravorty [24]. We emphasize here that the idempotent non-interacting one-matrix was used in all inequalities. We can, however, use the fact that the total kinetic energy is related with the non-interacting kinetic energy by $T \geq T_{s}$ [9], where the total interacting kinetic energy $T$ is given by a relation as Eq.(15) with the difference that the exact interacting one-matrix is applied instead of the idempotent non-interacting one-matrix. Therefore from inequality (23) one can readily obtain an equality for the total interacting kinetic energy $T$. However, it would be less sharper than (24).

Now, the relationship between the newly defined Shannon information $S_{F}$ and the usual Shannon information $S_{n}$ coming from the electron density is explored. Introduce the function $f$ with the definition

$$
\gamma\left(\mathbf{r}, \mathbf{r}^{\prime}\right)=n^{1 / 2}(\mathbf{r}) n^{1 / 2}\left(\mathbf{r}^{\prime}\right) f\left(\mathbf{r}, \mathbf{r}^{\prime}\right) .
$$

It can readly seen that $f(\mathbf{r}, \mathbf{r})=1$. From the idempotency (Eq. (8)) follows that

$$
\int n\left(\mathbf{r}_{2}\right) f^{2}\left(\mathbf{r}, \mathbf{r}_{2}\right) d \mathbf{r}_{2}=2
$$

for any $\mathbf{r}_{1}$. Substituting Eq. (25) into Eq. (9) and Eq. (11) we obtain

$$
S_{F}=\frac{2}{N} S_{n}+2 \ln N-\frac{1}{2 N} \int f^{2}(\mathbf{R}) \ln f^{2}(\mathbf{R}) d \mathbf{R} .
$$

For a one-level, two-electron system $f\left(\mathbf{r}, \mathbf{r}^{\prime}\right)=1$ and this relation reduces to Eq. (45). This case is studied in Section 4. 


\section{THE LOCAL WAVE-VECTOR, LINK BETWEEN THE SHANNON AND FISHER INFORMATION}

Following Nagy, Romera and Liu $[11,12]$ the local wave-vector is defined as

$$
\mathbf{q}(\mathbf{R})=-\frac{\nabla_{R} F(\mathbf{R})}{F(\mathbf{R})}
$$

It can also be written as

$$
\mathbf{q}(\mathbf{R})=-\nabla_{R}(\ln F)
$$

Eqs. (13),(14),(28) and (29) lead to the relations

$$
\mathbf{q}=\nabla_{R}\left(\frac{s_{F}}{F}\right)
$$

and

$$
q^{2}=\frac{i_{F}}{F}
$$

That is, the local wave-vector $\mathbf{q}$ provides a link between the Shannon and Fisher information: the local wave-vector is the gradient of the Shannon information per particle and the square of the local wave-vector is the Fisher information per particle. From Eqs. (20) and (31) follows the relationship between the local kinetic energy and local Fisher information:

$$
\tau_{s}=\frac{N}{16} F q^{2}
$$

while, the non-interacting kinetic energy takes the form

$$
T_{s}=\frac{N}{16} \int F q^{2} d \mathbf{R}
$$

that is, the non-interacting kinetic energy is proportional to the average of the square of the wave vector.

\section{RESULTS FOR COULOMB SYSTEMS}

We illustrate the results above by Coulomb systems. Consider first the case when the $\gamma$ has the form

$$
\gamma=A \exp \left[-a\left(r_{1}+r_{2}\right)\right]
$$

and consequently $F$ can be written

$$
F=\frac{A^{2}}{2 N} \exp \left[-2 a\left(r_{1}+r_{2}\right)\right]
$$

Expressions (34) and (35) are valid for the asymptotic region $\left(r_{1} \rightarrow \infty\right.$ and $\left.r_{2} \rightarrow \infty\right)$ for any system in Coulomb external potential $(v(r)=-Z / r)$. Eq. (35) gives the asymptotic behaviour of the distribution function $F$ with $a=\sqrt{2 I}$, where $I$ is the first ionization potential.

The Shannon and the Fisher information densities then take the form

$$
s_{F}=-F\left[2 \ln A-\ln (2 N)-2 a\left(r_{1}+r_{2}\right)\right]
$$

and

$$
i_{F}=a^{2} F=\frac{16}{N} \tau_{s}
$$

respectively.

$$
\frac{s_{F}}{F}=-\ln \tau_{s}+\ln N a^{2}-\ln 2
$$


The square of the local wave vector is

$$
q^{2}=8 a^{2}=\frac{i_{F}}{F}=\frac{16}{N} \frac{\tau_{s}}{F}
$$

Consider, now, a zero-order approximation to the one-matrix for the He isoelectronic series [25] :

$$
\gamma=\frac{Z-\alpha}{\pi} \exp \left[-(Z-\alpha)\left(r_{1}+r_{2}\right)\right]
$$

We are led to the Shannon information

$$
S_{F}=-6 \ln (Z-\alpha)+6+2 \ln \pi
$$

that can also be written as

$$
S_{F}=-3 \ln T_{s}-3 \ln 2+6+2 \ln \pi
$$

We mention here that Massen and Panos [26] proposed a relationship between the phase-space Shannon information and the kinetic energy.

The idempotent one-matrix for the He isoelectronic series can be written as :

$$
\gamma=2 \phi_{1 s}\left(r_{1}\right) \phi_{1 s}\left(r_{2}\right)
$$

where $\phi_{1 s}$ is the one-electron orbital. The Shannon information has the the form

$$
S_{F}=-2 \int n_{1 s}(r) \ln \left(n_{1 s}(r)\right) d \mathbf{r}
$$

where $n_{1 s}=\left(\phi_{1 s}\right)^{2}$ is the one-electron density. The Shannon information $S_{n}$ constructed from the electron density $n$ (Eq. (5) can be related to the $S_{F}$ :

$$
S_{F}=S_{n}+2 \ln 2
$$

Fig. 1 presents $S_{n}$ and $T_{s}$. $S_{n}$ has been numerically obtained using the analytical Hartree-Fock wave functions of Koga et al. [27] for the He isoelectronic series $(Z=2-9)$. The values of $T_{s}$ have been obtained from reference [28]. We have fitted these values obtaining (with a c. c. of 0.95 ) that

$$
S_{n}=-3.07 \ln T_{s}+7.25
$$

in good agreement with Eq. (42) that can be written in terms of $S_{n}$ as

$$
S_{n}=-3 \ln T_{s}+6.90 \text {. }
$$

Eq. (45) is valid only for a one-level, two-electron system. In other cases Eq. (27) should be used and the relationship between $S_{n}$ and $T_{s}$ cannot be described by expressions (46) or (47). It will be the subject of further research. Finally, we can utilize expression for the lower bound of $S_{n}$ derived by Gadre and Bendale [18]:

$$
S_{n} \geq 3 N(1+\ln \pi) / 2+(N \ln N) / 2-3 N \ln \left(3 N / 4 T_{s}\right) / 2 .
$$

Substituting $T_{s}$ from Eq. (19) into Eq. (48) the inequality for $S_{n}$ is expressed by the Fisher information $I_{F}$ :

$$
S_{n} \geq 3 N(1+\ln \pi) / 2+(N \ln N) / 2-3 N \ln \left(N / 12 I_{F}\right) / 2 .
$$

In summary, a six-dimentional distribution function is constructed from the one-matrix of a non-interacting system. For double occupied orbitals and real one-matrix, the Fisher information obtaineded from the six-dimentional distribution function is proportional to the non-interacting kinetic energy. A local six-dimensional wave-vector $q$ is defined that establishes a link between the Shannon and Fisher information: the local wave-vector is the gradient of the Shannon information per particle and the square of the local wave-vector is the Fisher information per particle. A relationship is explored between the non-interacting kinetic energy and the local wave-vector. 


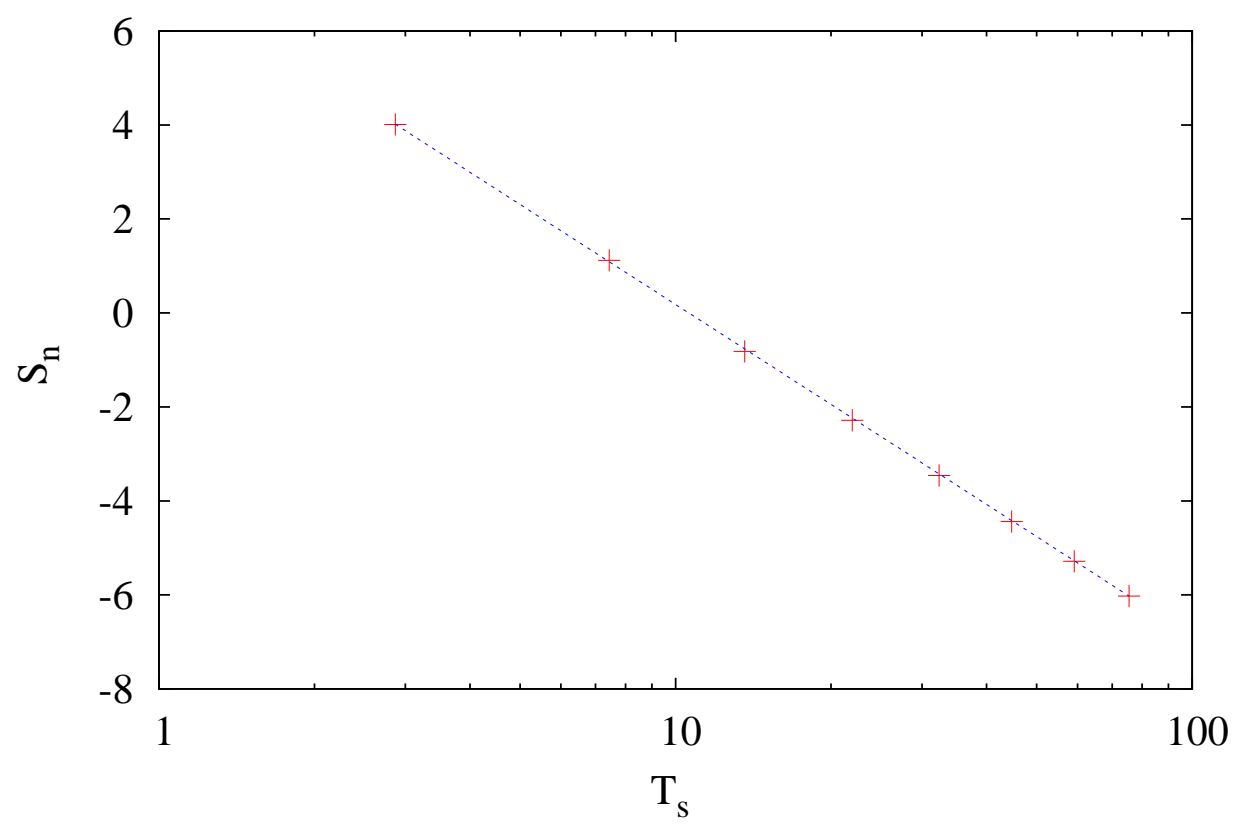

FIG. 1: $S_{n}$ in terms of $T_{s}$ for He-like ions. The crosses correspond to the Hartree-Fock values for the ions with nuclear charge $Z=2-9$. The dashed line corresponds to equation (46). All the data are in atomic units.

\section{Acknowledgments}

This work was supported by the Projects: FIS2011-24149 (Spanish MICINN). The work is also supported by the TAMOP 4.2.2.A-11/1/KONV-2012-0036 project. The project is co-financed by the European Union and the European Social Fund. Grant OTKA No. K 100590 is also gratefully acknowledged.

\section{References}

[1] S. B. Sears, R. G. Parr and U. Dinur, Israel J. Chem. 19, 165 (1980).

[2] R. A. Fisher, Proc. Cambridge Philos. Soc.22, 700 (1925).

[3] C. F. Weizsäcker, Z. Phys. 96341 (1935).

[4] Á. Nagy, J. Chem. Phys. 119, 9401 (2003).

[5] E. Romera, P. Sánchez-Morena and J. S. Dehesa, Chem. Phys. Lett. 414468 (2005).

[6] Á. Nagy, Chem. Phys. Lett.425, 157 (2006).

[7] E. Romera and J. S. Dehesa, J. Chem. Phys. 120, 8906 (2004).

[8] E. Romera, Mol. Phys. 100, 3325 (2002).

[9] M. Levy and J. P. Perdew, in Density Functional Methods in Physics, ed. R. M. Dreizler and J. da Prividencia, Plenum,New York, 1985, pp 11.

[10] Á. Nagy, Chem. Phys. Lett. 449, 212 (2007).

[11] Á. Nagy and S. B. Liu, Phys. Lett. A 3721654 (2008).

[12] Á. Nagy, E. Romera and S. B. Liu, Phys. Lett. A 377286 (2013).

[13] I. M. Ghiringhelli, L. P. Hamilton and L. Delle Site, J. Chem. Phys. 132, 014106 (2010).

[14] C. E. Shannon, Bell Syst. Tech. J. 27, 379 (1948).

[15] I. Bialynicki-Birula and J. Mycielski, Commun. Math. Phys. 44, 129 (1975). 
[16] S. R. Gadre, Phys. Rev. A 30, 620 (1984), S. R. Gadre and R. J. Bendale, Int. J. Quantum Chem. 28, 311 (1985).

[17] S. R. Gadre, S. B. Sears, S. J. Chakravorty and R. J. Bendale, Phys. Rev. A 32, 2602 (1985).

[18] S. R. Gadre and R. J. Bendale, Phys. Rev. A 36, 1932 (1987).

[19] S. B. Liu, J. Chem. Phys. 126, 191107 (2007); 126, 244103 (2007).

[20] Á. Nagy and K. D. Sen, Acta Physica Debrecina 45105 (2011).

[21] Á. Nagy and E. Romera, Chem. Phys. Lett. 490, 242 (2010).

[22] D. Chakraborty and P. W. Ayers, J. Math. Chem. 491810 (2011).

[23] E. Romera Mol. Phys. 100, 3325 (2002).

[24] S. R. Gadre and S. J. Chakravorty, J. Chem. Phys. 84, 7051 (1986).

[25] C. Schwartz, Ann. Phys. (N. Y. ) 6156 (1959).

[26] S. E. Massen and C. P. Panos, Phys. Lett. A 28065 (2001).

[27] T. Koga, M. Omura, H. Teruya and A. J. Thakkar, J. Phys. B 28, 3113 (1995).

[28] Q. Zhao and R. G. Parr, Phys. Rev. A 46, 2337 (1992). 